\title{
PREOPERATIVE EVALUATION OF BASAL FREE TRIIODOTHYRONINE IN PATIENTS UNDERGOING CORONARY ARTERY BYPASS GRAFTING SURGERY. DOES IT HELP?
}

\author{
Kaushal Kishore Tiwari ${ }^{1,2}$, Alfredo Giuseppe Cerillo ${ }^{1}$, Simona Storti ${ }^{3}$, Stefano Bevilacqua ${ }^{1}$, Aldo \\ Clerico $^{3}$, Giorgio Iervasi ${ }^{3}$, Mattia Glauber ${ }^{1}$ \\ ${ }^{1}$ Department of Cardiac Surgery, FTGM "G. Pasquinucci" Heart Hospital, Massa, Italy \\ ${ }^{2}$ Department of CTVS, College of Medical Sciences, Teaching hospital, Bharatpur, Nepal \\ ${ }^{3}$ Department of Clinical Laboratories, FTGM “G. Pasquinucci” Heart Hospital, Massa, Italy
}

Correspondence
Dr Kaushal K Tiwari
College of Medical Sciences,
Teaching Hospital
Bharatpur-10, Chitwan, Nepal
Email:
drkaushalkt@yahoo.com

DOI: http://dx.doi.org/10.3126/ jcmsn.v11i2.13668

\begin{abstract}
Background \& Objectives: The postoperative Low T3 syndrome has been considered as a possible source of reduced myocardial contractility, resulting in increased mortality after CABG. Effect of preoperative Low $\mathrm{T} 3$ has not been well studied in patients undergoing CABG surgery. Aim of our study is to evaluate effect of preoperative Low T3 syndrome in patients undergoing CABG surgery. Materials \& Methods: Six hundred and six patients undergoing $\mathrm{CABG}$ were included in this prospective study. The impact of the base-line FT3 concentration and of preoperative low T3 syndrome on the risk of postoperative low cardiac output and hospital death was analyzed. Results: Fifteen patients $(2.3 \%)$ postoperatively and $159(26.2 \%)$ developed major complications. At univariate analysis a reduced $\mathrm{EF}$, the presence of peripheral vascular disease, the NYHA class, the surgical urgency, the aortic cross-clamp time, the CPB time and the FT3 concentration at admission were significantly associated with low $\mathrm{CO}$ and higher mortality. At multivariate analysis, the CPB time, an emergency procedure, a reduced LVEF, and the fT3 concentration were independently related to the development of low CO. However, in multivariate analysis low EF, and the fT3 concentration were the only predictors of hospital death. Conclusion: We conclude that preoperative low EF and low T3 syndrome independently causes low cardiac output and higher mortality in patients undergoing CABG. Therefore, all patients undergoing CABG should be evaluated for low T3 syndrome and patients with low T3 syndrome should be considered at increased risk. Appropriate preoperative T3 replacement therapy could decrease the postoperative complications in patients undergoing $\mathrm{CABG}$.
\end{abstract}

Key words: $\mathrm{CABG}$, Euthyroid Sick Syndrome, Low T3 Syndrome.

Citation: Tiwari KK, Cerillo AG, Storti S, Bevilacqua S, Clerico A, Iervasi G, et al. Preoperative evaluation of basal free triiodothyronine in patients undergoing coronary artery bypass grafting surgery. Does it help? JCMS Nepal. 2015;11(2):1-7.

\section{INTRODUCTION}

Cardiac patients frequently present with a typical pattern of altered thyroid hormone metabolism, characterized by low circulating free triiodothyronine (FT3) levels in the absence of an intrinsic thyroid disease. This condition, variously known as the "Low T3 syndrome" (LT3S), the "Non-thyroidal illness syndrome" or the "Euthyroid sick syndrome" is mainly due to the reduced peripheral conversion of $\mathrm{T} 4$ into $\mathrm{T} 3$, and has been observed in patients with myocardial infarction, heart failure, and is almost invariably present after cardiac surgery with and without cardiopulmonary bypass. $^{2,3}$
The postoperative LT3S has been considered as a possible source of reduced myocardial contractility, resulting in increased morbidity and mortality after $\mathrm{CABG}^{1}$. Previous studies have shown that the postoperative LT3S after CABG surgery may have variable severity and duration ${ }^{2}$, but a correlation between the severity of the postoperative T3 deficiency, the postoperative myocardial function, and the clinical outcome has, to our knowledge, never been shown. Based on the observation that "There are similarities between hypothyroid patients and those undergoing cardiac surgery with respect to both serum triiodothyronine concentrations and decreased cardiac contractility 
and elevated peripheral vascular resistance", several well designed, and randomized protocols of substitutive therapy with IV T3 for patients undergoing $\mathrm{CABG}$ were developed during the nineties. ${ }^{4,5}$ Unfortunately, after the initial success of IV T3 as a rescue therapy in a small pilot study, ${ }^{5}$ the results of these trials showed only marginal benefits for the treated patients. ${ }^{1}$

However, the thyroid hormone has a strong influence on the expression of several structural and regulatory proteins of the cardiac myocyte, and the thyroid hormone regulated transcription is essential to maintain the physiological cardiac phenotype. ${ }^{1}$ For this reason, the T3 deficiency has been proposed as a causative factor in the pathophysiology of heart failure. ${ }^{1,6}$ Moreover, the presence of the LT3S at hospital admission has been definitively shown to be associated with increased mortality in the cardiac, non surgical patient. A relatively prolonged status of reduced $\mathrm{T} 3$ level, which can be frequently observed in patients coming to the hospital for a heart operation, could therefore provoke important alterations of the cardiac protein gene expression, ${ }^{6}$ resulting in increased cardiovascular vulnerability. All these facts led us to hypothesize that the presence of a low T3 syndrome prior to the operation could be associated with a worse postoperative course and with increased mortality in patients undergoing CABG surgery.

The present study was designed to identify preoperative and procedural variables predicting a more severe postoperative LT3S after CABG surgery; to evaluate the impact of "severe postoperative LT3S" on the postoperative course; and to analyze the prognostic role of the preoperative LT3S in the CABG patient's population.

\section{MATERIALS AND METHODS \\ Patients}

The impact of the base-line FT3 concentration and of preoperative low T3 syndrome (defined as an FT3 concentartion $<2.23 \mathrm{pmol} / \mathrm{l}$ ) on the risk of postoperative low cardiac output (low $\mathrm{CO}$ ) and hospital death was prospectively analyzed in 606 consecutive patients undergoing $\mathrm{CABG}$ at our institution from 1st October 2010, to 31st March 2012. The following end points were considered: postoperative complications; severe complications (any life-threatening complication requiring major medical or surgical treatment); need for inotropes (dopamine or dobutamine for $\geq 24 \mathrm{~h}$ ); low $\mathrm{CO}$ (requirement for more than one of the above mentioned drugs or for adrenaline, noradrenaline, enoximone, or IABP); and hospital death (death after surgery in the hospital or in a rehabilitation facility without prior discharge at home, or any death occurring $\leq 30$ days after surgery).

In the first 303 patients (Subgroup A), the thyroid profile was also evaluated immediately after the end of the operation (T0), and then at 12, 48, and 120 hours postoperatively. These time points were identified as relevant from the analysis of data collected on a smaller population at 14 different time points in a previous study (CER ATS). In this subset of patients, the severity of the postoperative LT3S was assessed by the time course of the FT3 concentration over time: the LT3S was defined severe when at 120 hours postoperatively the FT3 concentration was still reduced below the low normal limit, and did not show a trend towards improvement (Figure 1). The sum of the single FT3 concentration values for each patient from T0 to T120 ("Total postoperative FT3", TPT3), was also used as a marker of severity of the postoperative LT3S.

Patients with a known or clinically evident thyroid disease, and patients taking drugs known to interfere with thyroid function were excluded. The presence of any predominant severe systemic disease was also considered as exclusion criteria. This study was approved by the Ethical Committee of the Fondazione G. Monasterio, G. Paquinucci Heart Hospital, Massa, Italy. Informed consent was obtained from all patients.

\section{Thyroid hormone sampling}

The thyroid profile is routinely studied as a part of the baseline, preoperative evaluation at our Institution. At admission, $5 \mathrm{ml}$ blood sample taken from all patients. Four additional $5 \mathrm{ml}$ blood samples were collected in the subgroup A patients. All samples were collected in serum separator tubes and immediately centrifuged and analyzed. For the present study, the free T3 (fT3), free thyroxine (fT4) and thyroid stimulating hormone (TSH) were assayed on all samples with the AxSYMw Microparticle Enzyme Immunoassay (MEIA, Abbott Laboratories, Diagnostic Division, Rome, Italy). In our laboratory, the mean intra- and interassay coefficients of variation and the sensitivity were, respectively: $4 \%, 5.15 \%$ and 0.012 $\mathrm{mIU} / \mathrm{mL}$ for TSH; $2.67 \%, 1.18 \%$ and $0.017 \mathrm{pmol} / \mathrm{L}$ for FT3; $4 \%, 2.8 \%$ and $0.3 \mathrm{ng} / \mathrm{mL}$ for T3; $1.93 \%$, $1.7 \%$ and $5.2 \mathrm{pmol} / \mathrm{L}$ for FT4; $1.6 \%, 1.83 \%$ and $1.05 \mathrm{mg} / \mathrm{dL}$ for $\mathrm{T} 4$; and $1.53 \%$ and $1.18 \%$ for Tuptake. The reference intervals for our laboratory are as follows: T3 $-0.45-1.37 \mathrm{ng} / \mathrm{mL}$; T4 $-4.5-12$ $\mathrm{mg} / \mathrm{mL}$; FT3 - 2.23-5.35 pmol/L; FT4 - 9.14-23.81 $\mathrm{pmol} / \mathrm{L}$; TSH - 0.47-4.64 mIU/mL; and T-uptake $0.66-1.27$. 


\section{Anesthetic technique and surgical management}

The anesthetic technique and surgical management was according to the institutional protocol. Total intravenous anesthesia with diazepam, fentanyl, pancuronium and propofol was used in all cases. The decision in regards to on pump or off pump CABG was left to the operating surgeon, and was mostly based on the severity and extension of disease of the target vessels. Cardiopulmonary bypass (CPB) was conducted on moderate hypothermia $\left(34^{\circ} \mathrm{C}\right)$, and myocardial protection was achieved by using intermittent anterograde hyperkalemic warm blood cardioplegia. The final goal of CABG surgery was to obtain a complete myocardial revascularization.

\section{Statistical analysis}

Continuous variables are expressed as mean \pm standard deviation. Dichotomous variables are expressed as percentages. The effect of baseline patient's characteristics and of procedural and postoperative variables on the postoperative course of the FT3 concentration was analyzed by the ANOVA for repeated measures. The association of preoperative, intraoperative and postoperative variables, including the postoperative LT3S severity and the TPT3, with postoperative low CO and hospital death was investigated by the Fisher's exact test (dichotomous variables), or by the unpaired Student's t-test (continuous variables). Non-normally distributed continuous variables were analyzed by the Mann-Whitney U-test. The tested variables are listed and defined in the Appendix A. Factors significantly influencing the end point of the study were then included in a logistic multivariate regression model to ascertain their independent role. Also factors for which the univariate analysis gave a $\mathrm{P}$ value $\leq 0.1$, or of known biologic significance, but failing to meet the critical $\alpha$ level, were included. Odds ratio (OR) and 95\% confidence interval (CI) were calculated. Receiver operating characteristic (ROC) curves were calculated to single out the best cutoff value of fT3 predicting postoperative low CO and hospital death. The accuracy of the test was assessed measuring the area under the ROC curve (AUC). The AUC was assessed by the non-parametric method of DeLong and Clarke-Pearson. The nonsymmetric $95 \%$ CI for AUC was computed using the Bootstrap Percentile Method, with 1000 bootstrap replications. The statistical significance of difference of AUC from that of the 'line of no information' was evaluated by Mann-Whitney Ustatistic. A $\mathrm{P}$ value $<0.05$ was considered significant. Statistical analysis was conducted using
SPSS software version 10.1 (SPSS Inc, Chicago, IL).

\section{RESULT \\ Patients}

Six-hundred and six patients were included. Mean age was $67.3 \pm 10.4$ years, and $461(76.1 \%)$ patients were male. Mean left ventricle ejection fraction (LVEF) was $51 \pm 10 \%$, and 104(17.1\%) patients had a LVEF $\leq 40 \%$. Most patients $(63.7 \%)$ had three vessels disease. Mean fT3 at admission was $3.3 \pm 0.9 \mathrm{pmol} / \mathrm{l}$, mean $\mathrm{fT} 4$ was $13.9 \pm 2.8 \mathrm{pmol} / \mathrm{l}$, and mean TSH was $2.1 \pm 2.4 \mathrm{mIU} / \mathrm{ml}$. Sixty-five patients $(10.7 \%)$ had a low T3 syndrome (defined as a fT 3 concentration $\leq 2.23 \mathrm{pmol} / 1$ in the absence of an intrinsic thyroid disease) at admission. Demographic data are presented in Table 1.

Table 1. Demographic data

\begin{tabular}{|ll}
\hline Factors & Measurements \\
fT3 (pmol/l) & $3.3 \pm 0.93$ \\
fT4 (pmol/l) & $13.91 \pm 2.85$ \\
\hline TSH mIU/ml & $2.1 \pm 2.4$ \\
\hline Age, years & $67.3 \pm 10.4$ \\
\hline Sex (M, \%) & $76.1 \%$ \\
\hline Hypertension, n (\%) & $460(75.9 \%)$ \\
\hline Diabetes, n (\%) & $185(30.5 \%)$ \\
\hline Smoker, n (\%) & $371(61.2 \%)$ \\
\hline Dyslipidemia, n (\%) & $415(68.4 \%)$ \\
\hline LVEF \% & $51 \pm 10$ \\
\hline 3 vessel disease, (\%) & $63.7 \%$ \\
\hline COPD, n (\%) & $91(15.01 \%)$ \\
\hline Peripheral vascular disease, $\mathrm{n}(\%)$ & $88(14.5 \%)$ \\
\hline CPB time (min) & $94.2 \pm 48.8$ \\
\hline Aortic Cross clamp time (min) & $57.2 \pm 33.4$ \\
\hline
\end{tabular}

\section{Early outcome and complications}

Fifteen patients $(2.4 \%)$ died postoperatively. The complications observed in these patients and the causes of death are reported in Table 2 .

Twenty-seven (4.4\%) patients had myocardial ischemia postoperatively. Eighteen of these $(2.9 \%)$ developed a perioperative myocardial infarction (POMI). Sixty-four $(10.5 \%)$ patients needed inotropic support for $\geq 24 \mathrm{~h}$, and $31(5.1 \%)$ had severe low output syndrome. Other postoperative complications included major bleeding in 34(5.6\%), respiratory failure in $49(8.1 \%)$, acute renal failure in $49(8.1 \%)$, and cerebro vascular accident $(1.1 \%)$ (Table 2). Mean postoperative ICU stay was $28.9 \pm$ $7.4 \mathrm{~h}$. Mean postoperative hospital stay, including cardio-respiratory rehabilitation when needed, was $8.9 \pm 3.6$ days (Table 2 ). 
Postoperative course of the thyroid hormones (Subgroup A patients)

The FT3 concentration dropped significantly after the operation, and remained low throughout the study period $(p<0.0001) \quad$ (Figure 2). The postoperative FT3 course was not influenced by any of the tested preoperative or procedural variables (Figure 3).

Table 2. Postoperative data

\begin{tabular}{ll} 
Factors & Measurements \\
\hline Post operative MI, n (\%) & $18(2.9 \%)$ \\
Bleeding, n (\%) & $34(5.6 \%)$ \\
\hline Inotropes use, n (\%) & $64(10.5 \%)$ \\
Respiratory insufficiency, n (\%) & $49(8.1 \%)$ \\
Acute renal failure, n (\%) & $49(8.1 \%)$ \\
ICU stay (hours) & $28.9 \pm 7.4$ \\
Mechanical ventilation (hr) & $11 \pm 18$ \\
Postoperative ward stay (days) & $8.9 \pm 3.6$ \\
\hline Postoperative mortality, n (\%) & $15(2.4 \%)$
\end{tabular}

The postoperative course of FT4, Total T3, Total T4 and TSH is reported in figure 2. A severe postoperative LT3S was present in 241 out of 303 (79.5\%) patients. The severity of the postoperative LT3S was not associated with an increased rate of complications $(p=0.82)$, severe complications $(p=0.73)$, need for inotropes $(p=0.70)$, low output syndrome $(\mathrm{p}=0.84)$, or death $(\mathrm{p}=0.97)$. Of note, in the 241 patients showing a "type B" curve (Figure 1), the FT3 concentration was still reduced well below our laboratory's low normal limit on postoperative day $5(1.91 \pm 0.05 \mathrm{pmol} / \mathrm{l})$, indicating that surgery induced a marked alteration of the

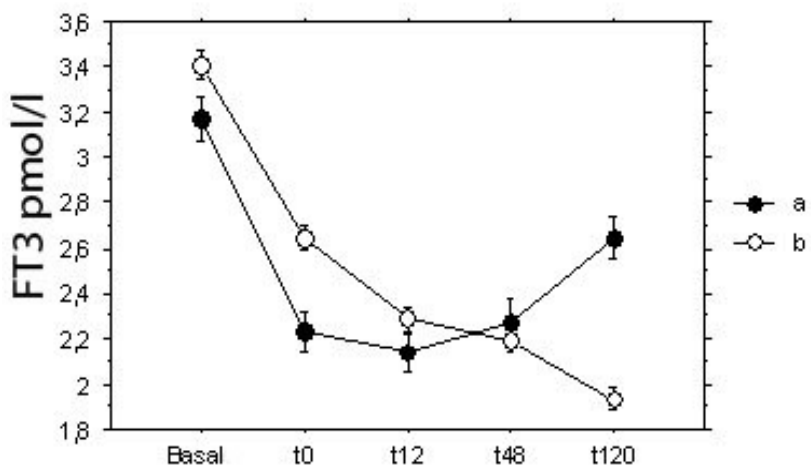

Figure 1. To evaluate the possible detrimental effect of a severe and prolonged postoperative reduction of the FT3 concentrations, patients were divided in two groups: group a included 62 patients in whom, after an initial drop of the FT3 concentration, the thyroid function had returned to normal by the postoperative day 5 . Group $b$ included 242 patients in whom the FT3 concentration declined further, and reached the lower value $(1.91 \pm 0.05$ pmol/l) by postoperative day 5 .
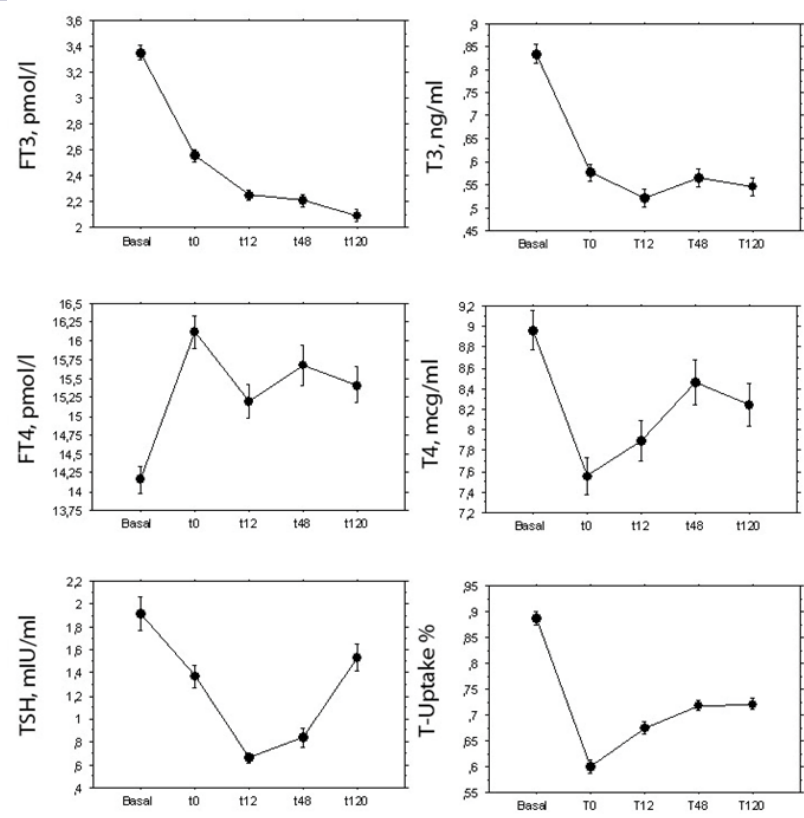

Figure 2. Postoperative course of the FT3, T3, FT4, T4, TSH concentration and of T-Uptake.

hypothalamus-pituitary-thyroid axis function in most patients.

The reverse analysis was also carried out, to evaluate the possible influence of postoperative complications on the time course of the FT3 concentration. The results of this analysis are detailed in Figure 3. The FT3 concentration was not apparently in relation with the occurrence of low cardiac output (Figure 3a, 3b) and of severe complications or death (Figure 3c, 3d). However, patients experiencing at least one complication (including minor complications) had a more marked reduction of the FT3 levels postoperatively $(\mathrm{p}=0.02$, Figure 3e).

Similarly, a lower TPT3 was not associated with an increased incidence of postoperative low cardiac output $(\mathrm{p}=0.54)$ and death $(\mathrm{p}=0.12)$. However, a reduced TPT3 was more frequent in patients experiencing postoperative complications $(\mathrm{p}=0.0001)$, and severe complications $(\mathrm{p}=0.02)$.

\section{Predictors of low cardiac output and death}

As already stated, 64 patients (10.5\%) needed inotropic support postoperatively, and of these 31 (5.1\%) developed severe low CO. At univariate analysis a reduced EF, an EF lower than $40 \%$, the presence of peripheral vascular disease, the NYHA class, the surgical priority, the aortic cross-clamp time, the cardiopulmonary bypass time and the FT3 concentration at admission were significantly associated with the development of low CO. At multivariate analysis, the cardiopulmonary bypass time $(\mathrm{OR}=1.025 ; 95 \% \mathrm{CI}: 1.0-1.04 ; \mathrm{p}=0.003)$, 

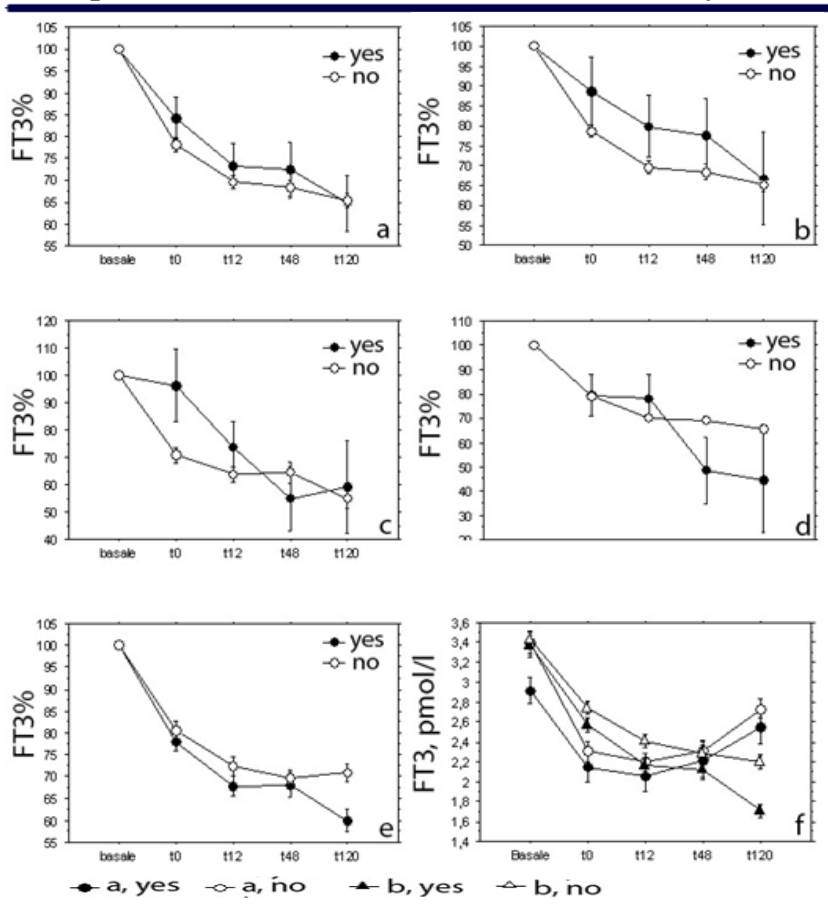

Figure 3. The postoperative percent reduction of the FT3 concentration in complicated vs uncomplicated patients. (a) postoperative need for inotropic support $(\mathrm{p}=0.42)$; (b) postoperative low cardiac output $(\mathrm{p}=0.37)$; (c) severe complication $(p=0.34)$; hospital death $(p=0.09)$; any complication $(\mathrm{p}=0.02)$.(e) Impact of postoperative complications on the postoperative course of the thyroid hormone in patients with a mild (A) and severe (B) postoperative low $\mathrm{T} 3$ syndrome. In patients with a mild postoperative LT3S, complications were more likely when the FT3 at admission was lower $(p=0.006)$. Furthermore, the occurrence of complications was associated with a more pronounced decline of the FT3 concentration by the postoperative day 5 in the group B patients $(p=0.006)$, but not in the group A $(p=0.16)$.

an emergency procedure $(\mathrm{OR}=10.153 ; 95 \% \mathrm{CI}: 3.1$ $-32.8 ; \mathrm{p}=0.0001)$, a reduced LVEF $(\mathrm{OR}=0.917$; 95\%CI: $0.84-0.99 ; p=0.03)$, and the fT3 concentration $(\mathrm{OR}=0.371 ; 95 \% \mathrm{CI}$ : $0.2-0.68 ; \mathrm{p}=$ $0.001)$ were independently related to the development of low CO.

Fifteen patients died, and the overall 30 days mortality was $2.4 \%$. At univariate analysis, the left ventricular $\mathrm{EF}$, the surgical priority, the cardiopulmonary bypass time, and the fT3 concentration at admission were significantly associated with death. The presence of chronic obstructive pulmonary disease was also included in the multivariate analysis, both for its well known negative prognostic value in the CABG patient population, and because univariate analysis showed an evident trend toward statistical significance (OR $=3.749 ; 95 \% \mathrm{CI}: 1.08-12.9 ; \mathrm{p}=0.0596)$. At multivariate analysis, a low EF (OR $=0.941 ; 95 \%$ CI: $0.9-0.984 ; p=0.008)$, and the fT3 concentration $(\mathrm{OR}=0.32 ; 95 \% \mathrm{CI}: 0.15-0.68 ; \mathrm{p}=$ $0.003)$ were the only predictors of hospital death. Of note, in our series a base-line FT3 concentration $<2.23 \mathrm{pmol} / 1$ was associated with a tenfold increase of the risk of postoperative low $\mathrm{CO}$ (OR 11.511; 95\%CI: $5.4-24.7 ; \mathrm{p}=0.0001)$, and death (OR 10.714; 95\%CI: $3.7-30.6 ; \mathrm{p}=0.0001$ ), and with longer time on mechanical ventilation (18.5 \pm 4.0 vs $8.4 \pm 0.7$ hours, $\mathrm{p}=0.001$ ), longer ICU stay $(65.5 \pm 23.4$ vs $22.8 \pm 1.5$ hours, 0.0003$)$ and longer postoperative hospital stay $(11.1 \pm 1.2$ vs $8.4 \pm 0.2$ days, $\mathrm{p}=0.07$ ).

According to the ROC curve analysis, the best threshold value of fT3 for predicting the occurrence of postoperative low CO was $2.82 \mathrm{pmol} / 1$ (ROC area, 0.774; 95\% CI 0.739- 0.807; $\mathrm{P}<0.0001)$. This cutoff value showed a sensitivity of 0.727 and a specificity of 0.755 . The low normal cutoff value of our laboratory for the FT3 concentration (2.23 pmol/l) showed a sensibility of 0.515 and a specificity of 0.904 . The best threshold value of fT3 for predicting death was $2.39 \mathrm{pmol} / 1$ (ROC area, $0.781 ; 95 \%$ CI $0.746-0.813$; P 0.0001 ). This cutoff value showed a sensitivity of 0.6 and a specificity of 0.88 .

\section{DISCUSSION}

The thyroid hormone exerts deep influence on the cardiovascular homeostasis, through both genomic and non-genomic mediated effects. ${ }^{1}$ Therefore, the low T3 syndrome has been established as a strong predictor of death in the cardiac, non-surgical patients. ${ }^{3,7}$ Since cardiac surgery may bring about important alterations of the thyroid function, the postoperative low T3 syndrome has been the object of intense research during the last twenty years. The results of this study demonstrate that the presence of the low T3 syndrome at hospital admission is associated with a markedly increased risk of postoperative myocardial dysfunction and death in patients undergoing CABG surgery. In our series, patients with low $\mathrm{T} 3$ at admission required more inotropic drugs and were more prone to develop a postoperative low cardiac output syndrome, suggesting that the observed increase in the hospital mortality is at least in part explained by a reduced cardiac reserve.

A secondary finding of our study was that the severity of the postoperative low T3 syndrome did not apparently influence the postoperative course (figure 3). Instead, our data suggest that a marked alteration of the thyroid profile, lasting up to several days, is present in the vast majority of patients after CABG surgery, and that in its more severe forms, this postoperative low T3 syndrome may represent 
the consequence, rather than the cause, of postoperative complications and prolonged hospital stay.

Thyroid hormone and the heart: is the result of the present study biologically plausible?

Normal FT3 levels are essential for the maintenance of the physiologic cardiac myocyte phenotype. In an in vitro human model, Forini and coworkers demonstrated that $\mathrm{T} 3$ deprivation has detrimental effects on myocardial histology and morphology, leading to structural and functional abnormalities that closely resemble those observed in heart failure, including decreased expression of SERCA2 and of $\alpha$-sarcomeric-actinin. ${ }^{8}$ Furthermore, it has been demonstrated that the thyroid hormone is able to regulate the extracellular matrix synthesis. T3 administration has been shown to prevent myocardial fibrosis in aortic banded animals, and to regulate the collagen type I biosynthesis. ${ }^{7}$ Finally, thyroid hormone deficiency is known to be associated with important alterations of the coronary blood flow due to a rarefaction in the myocardial arterioles, and with ischemic myocite loss. ${ }^{7}$

In light of these experimental evidences, the hypothesis that the increased incidence of postoperative low cardiac output and death that we observed in patients with low T3 is at least in part due to molecular and structural alterations of the myocardium, related to the reduced fT3 availability, seems extremely likely.

This conclusion is further supported by several clinical studies. Patients with heart failure and low T3 have a lower cardiac index, a higher left ventricular end-diastolic pressure and a greater functional impairment assessed by the VO2 peak compared with patients with a normal thyroid profile. ${ }^{9}$ Moreover, there is convincing evidence that a low T3 status is associated with reduced survival in the cardiac patient, the overall mortality at one year was almost five times higher in patients with low T3 at admission. ${ }^{3}$ More recently, Iervasi and coworkers were able to demonstrate that, in a cohort of 3121 cardiac patients, the presence of a mild thyroid dysfunction (low T3 syndrome or subclinical hypothyroidism) was associated with increased long term mortality. ${ }^{8}$ The result of our study is in line with these previous observations, and represents the first reported demonstration of the fact that the low T3 syndrome has a negative impact on the outcome of cardiac surgery.

Low T3 syndrome and cardiac surgery: an innocent postoperative phenomenon or a strong preoperative risk factor?
Since cardiac surgery is a frequent cause of low T3 syndrome, previous research mainly focused on the potentially harmful effects of the postoperative low T3 syndrome provoked by the operation itself, and on the role of IV T3 in the management of postoperative pump failure. Novitzky and coauthors from the University of Cape Town Medical School administered T3 intravenously to ten patients with severe intraoperative or early postoperative low cardiac output. All ten patients showed haemodynamic improvement, were weaned from inotropic support, and made an uneventful postoperative recovery thereafter. ${ }^{5}$

In light of our results, this is not surprising: in the present series, almost $80 \%$ of the patients developed a severe postoperative low T3 syndrome, but only 64 out of 606 patients required some inotropic support, indicating that in most instances, the postoperative drop of the FT3 concentration had no measurable haemodynamic consequences. On the other hand, the presence of reduced FT3 levels preoperatively was associated with a tenfold increase of the risk of low $\mathrm{CO}$ and death. A possible explanation of this apparent paradox is that the alteration of the cardiac myocyte phenotype caused by the low T3 syndrome may need some time to develop, and is probably not present at 120 hours after the operation, while it could be fully expressed at the time of the operation in patients with preoperative LT3S.

\section{Limitations of the study}

We were not able to collect tissue samples for histology and molecular analysis in this patient series. Our pathophysiologic model was therefore only hypothesized and could not be tested in an experimental setting. However, a protocol involving molecular studies on ventricular myocardium specimens in a big number of patients undergoing a closed heart procedure would have raised important ethical issues. A molecular study on a smaller number of patients will be necessary to demonstrate that patients with low T3 at admission do have an altered cardiac myocyte phenotype. This notwithstanding, we are convinced that the experimental and clinical evidence accumulated in the last decade strongly supports our model.

\section{CONCLUSION}

Patients with preoperative low T3 syndrome undergoing $\mathrm{CABG}$ surgery are at higher risk of developing postoperative low cardiac output, and experience higher hospital mortality. Therefore, the thyroid profile should be evaluated in all the patients undergoing surgical myocardial 
revascularization, and patients with low T3 should be considered at increased risk. Appropriate T3 replacement therapy should be considered preoperatively in patients with low T3 to minimize the complications in patients undergoing CABG. Further studies are needed to elucidate the molecular basis of this increased vulnerability, and to evaluate the possible benefit of a targeted substitutive therapy protocol.

\section{REFERENCES}

1. Bello G, Ceaichisciuc I, Silva S, Antonelli M. The role of thyroid dysfunction in the critically ill: a review of the literature. Minerva Anestesiologica. 2010;76(11):919-28. PMID:20935602.

2. Cerillo AG, Sabatino L, Bevilacqua S, Farneti PA, Scarlattini M, Forini F, et al. Non-thyroidal illness syndrome in off-pump coronary artery bypass grafting. Ann Thorac Surg. 2003;75:82-7. DOI:10.1016/S0003-4975 (02)03919-X.

3. Iervasi G, Pingitore A, Landi P, Raciti M, Ripoli A, Scarlattini $M$, et al. Low-T3 Syndrome: a Strong Prognostic Predictor of Death in patients With Heart Disease. Circulation. 2003 Feb 11;107(5):708-13. DOI:10.1161/01.CIR.0000048124.64204.3F; PMID:12578873.

4. Klemperer JD, Klein I, Gomez M, Helm RE, Ojamaa K, Thomas SJ, et al. Thyroid hormone treatment after coronary-artery bypass surgery. N Engl J Med. 1995;333:1522-7. DOI:10.1056/NEJM199512073332302; PMID:7477166.

5. Novitzky D, Copper DKC, Barton CI, Greer A, Chaffin J, Grim J, et al. Triiodothyronine as an inotropic agent after open heart surgery. J Thorac Cardiovasc Surg. 1989;98:972 -8. PMID:2682025.

6. Bennett-Guerrero E, Jimenez JL, White WD, D'Amico EB, Baldwin BI, Schwinn DA, et al. Cardiovascular effects of intravenous triiodothyronine in patients undergoing coronary artery bypass graft surgery: a randomized, double -blind, placebo- controlled trial. Duke T3 Study group. JAMA. 1996;275:687-92. DOI:10.1001/jama.275.9.687; 10.1001/jama.1996.03530330031025; PMID:8594265.

7. Galli E, Pingitore A, Iervasi G. The role of thyroid hormone in the pathophysiology of heart failure: clinical evidence. Heart Fail Rev. 2010;15(2):155-69. DOI:10.1007/s10741-008-9126-6; PMID:19110971.

8. Iervasi G, Nicolini G. Thyroid hormone and cardiovascular system: from basic concept to clinical application. Intern Emerg Med. 2013 Apr;8 Suppl 1:S71-4. DOI:10.1007/ s11739-013-0911-4; PMID:23435988.

9. Forini F, Paolicchi A, Pizzorusso T, Ratto GM, Saviozzi M, Vanini V, et al. 3,5,3'-Triiodothyronine deprivation affects phenotype and intracellular ( $\mathrm{Ca} 2+) \mathrm{i}$ of human cardiomyocytes in culture. Cardiovasc Res. 2001 Aug 1;51 (2):322-30. DOI:10.1016/S0008-6363(01)00287-5.

10. Biondi B. Mechanism in endocrinology: Heart failure and thyroid function. Eur J Endocrinol. 2012 Nov;167(5):60918. DOI:10.1530/EJE-12-0627; PMID:22956554. 